\title{
CONVERGENCE OF THE BACKFITTING ALGORITHM FOR ADDITIVE MODELS
}

\author{
CRAIG F. ANSLEY and ROBERT KOHN
}

(Received 14 June 1991; revised 21 February 1992)

Communicated by A. J. Pakes

\begin{abstract}
The backfitting algorithm is an iterative procedure for fitting additive models in which, at each step, one component is estimated keeping the other components fixed, the algorithm proceeding component by component and iterating until convergence. Convergence of the algorithm has been studied by Buja, Hastie, and Tibshirani (1989). We give a simple, but more general, geometric proof of the convergence of the backfitting algorithm when the additive components are estimated by penalized least squares. Our treatment covers spline smoothers and structural time series models, and we give a full discussion of the degenerate case. Our proof is based on Halperin's (1962) generalization of von Neumann's alternating projection theorem.
\end{abstract}

1991 Mathematics subject classification (Amer. Math. Soc.): 62G05, 62J02, 65D07, 65D10.

Keywords and phrases: Additive model, altemating projection, concurvity, nonparametric regression, penalized least squares, partial spline, trend, seasonal.

\section{Introduction}

We consider a model where the observations are a sum of $m$ unknown functions plus noise and we wish to estimate the unknown functions by penalized least squares. Thus we have

$$
y(i)=\sum_{j=1}^{m} f_{i}\left(x_{i j}\right)+e(i)
$$

with the $x_{i j}, i=1, \ldots, n, j=1, \ldots, m$ being the design points and $e(i)$ the noise. Although it is usually very expensive computationally to simultaneously estimate $f_{1}, \ldots, f_{m}$, if we fix any $m-1$ of the components, then an $O(n)$ algorithm is generally available for estimating the remaining component. This suggests the

(c) 1994 Australian Mathematical Society 0263-6115/94 \$A2.00+0.00 
following iterative scheme for solving the penalized least squares problem. Starting with some initial estimates of $f_{1}, \ldots, f_{m}$ (possibly zero), estimate $f_{1}$ by penalized least squares holding $f_{2}, \ldots, f_{m}$ fixed at their current estimates, then estimate $f_{2}$ holding $f_{1}, f_{3}, \ldots, f_{m}$ fixed at their current estimates, and continue this process until the estimates converge. This iterative method for estimating unknown components of an additive model is called backfitting and was used by Friedman and Stuetzle (1981) for projection pursuit regression, and by Wecker and Ansley (1982) and Buja, Hastie and Tibshirani (1989) for fitting additive spline models. Buja et al. (1989) analyzed the backfitting algorithm for a class of important problems and showed that it converged both for the regular case where there is only one solution and also for the degenerate case where there are multiple solutions.

This paper provides a geometric approach to the backfitting method for a general class of penalized least squares problems by showing that the solution to a penalized least squares problem is a projection in an appropriate space $S$ say, and that backfitting corresponds to successive projections onto subspaces $M_{1}, \ldots, M_{m}$ whose intersection is $S$. Therefore, backfitting corresponds to the alternating projection method proposed by von Neumann (1950) and extended by Halperin (1962). We can therefore apply von Neumann's convergence result on alternating projections to deduce the convergence of the backfitting method to the solution of the penalized least squares problem. von Neumann's alternating projection method, generalized by Halperin (1962), can be described as follows.

THEOREM 1.1. (Halperin, 1962) Suppose that $H$ is a Hilbert space, $S$ is a subspace of $H$ and $P$ is the projection onto $S$. Let $M_{1}, \ldots, M_{m}$ be subspaces of $H$ so that $S=M_{1} \cap \cdots \cap M_{m}$, and let $T_{j}$ be the projection onto $M_{j}, j=1, \ldots, m$. Form $T=$ $T_{m} T_{m-1} \cdots T_{1}$. Then $T^{N}$ converges strongly to $P$ as $N \rightarrow \infty$; that is $T^{N}(f) \rightarrow P(f)$ as $N \rightarrow \infty$ for any $f \in H$.

The alternating projection method is useful when projection onto the space $S$ is difficult, but projecting onto the subspaces $M_{j}$ is relatively easy, so that the projection $P$ can be obtained by sequentially applying the projections $T_{j}$. This is true, in particular, for the additive model (1.1).

The key to our approach is to treat the residuals as an extra component, thus reducing the penalized least squares problem to an interpolation problem whose solution is a projection in an appropriately defined Hilbert space. Our treatment is more general than that in Buja et al. (1989) as we prove convergence of the backfitting algorithm not only at the design points $x_{i j}$ but also at all values of the arguments of the functions. In addition we allow the evaluation functionals $f_{j}\left(x_{i j}\right)$ to be replaced by general linear functionals. This generalization is of more than just theoretical interest. Two examples requiring this extra generality are cubic spline smoothing with a periodic component which is discussed in Example 2.2 and the estimation of an additive model 
with trend and seasonal components discussed in Example 3.1.

The main feature of our approach, however, is that it provides a geometric solution to penalized least squares making the existence, uniqueness and convergence results transparent. To simplify our discussion, we first give results for the non-degenerate case where there is a unique solution for each of the components. In Section 3 we extend the results to the degenerate case, where there may be multiple solutions. Buja et al. (1989) refer to this as the problem of concurvity. Related methods for the block iterative solution of equations are given by Kaczmarz (1937) and Elfving (1980).

\section{Nondegenerate Case}

Consider the following mathematical structure. Suppose that $F_{1}, \ldots, F_{m}$ are linear spaces and $\langle\cdot, \cdot\rangle_{0 j}$ is a semi-inner product on $F_{j}, j=1, \ldots, m$, with corresponding semi-norm $\|\cdot\|_{0_{j}}$. By a semi-inner product we mean that $\langle\cdot, \cdot\rangle_{0_{j}}$ is an inner product except that $\left\|f_{j}\right\|_{0 j}$ can be zero with $f_{j} \neq 0$. For $i=1, \ldots, n$, let $\gamma_{i j}$ be a linear functional in the space $F_{j}, j=1, \ldots, m$. Define $F$ as the product space $F=$ $F_{1} \otimes \cdots \otimes F_{m}$, with typical element $f=\left(f_{1}, \ldots, f_{m}\right)$ and for $i=1, \ldots, n$ let $\gamma_{i}(f)=\gamma_{i 1}\left(f_{1}\right)+\cdots+\gamma_{i m}\left(f_{m}\right)$. We will assume that

ASSUMPTION 2.1. For $f \in F$, if $\left\|f_{j}\right\|_{0_{j}}=0$ for $j=1, \ldots, m$ and $\gamma_{i}(f)=0$ for $i=1, \ldots, n$, then $f=0$.

Assumption 2.1 implies that

$$
\left\langle f_{j}, g_{j}\right\rangle_{j}=\left\langle f_{j}, g_{j}\right\rangle_{0 j}+\sum_{i=1}^{n} \gamma_{i j}\left(f_{j}\right) \gamma_{i j}\left(g_{j}\right), \quad f_{j}, g_{j} \in F_{j}
$$

is a proper inner product for $F_{j},(j=1, \ldots, m)$ and

$$
\langle f, g\rangle_{F}=\sum_{j=1}^{m}\left\langle f_{j}, g_{j}\right\rangle_{0 j}+\sum_{i=1}^{n} \gamma_{i}(f) \gamma_{i}(g), \quad f, g \in F
$$

is a proper inner product for $F$. Let $\left\|f_{j}\right\|_{j}=\left\langle f_{j}, f_{j}\right\rangle_{j}^{1 / 2}$ and $\|f\|_{F}=\langle f, f\rangle_{F}^{1 / 2}$ be the corresponding norms. We assume further that

ASSUMPTION 2.2. $F_{j}$ is a Hilbert space, that is, complete, under the inner product (2.1), $(j=1, \ldots, m)$ and $F$ is a Hilbert space with inner product (2.2).

We observe

$$
y(i)=\sum_{j=1}^{m} \gamma_{i j}\left(f_{j}\right)+e(i)=\gamma_{i}(f)+e(i) \quad(i=1, \ldots, n)
$$


where $f_{j} \in F_{j}$ and $e(i)$ is the unobserved noise. We propose to estimate the unobserved function $f_{1}, \ldots, f_{m}$ by the penalized least squares criterion

$$
\underset{f \in F}{\operatorname{minimum}} \sum_{i=1}^{n}\left\{y(i)-\gamma_{i}(f)\right\}^{2}+\sum_{j=1}^{m}\left\|f_{j}\right\|_{0 j}^{2} .
$$

EXAMPLE 2.1. Additive spline model. We consider the additive model (1.1) with the components $f_{1}, \ldots, f_{m}$ defined on the interval $[0,1]$. Let $F_{A}$ be the space of functions on $[0,1]$ having square integrable second derivatives. Let $X$ be the $n \times m$ matrix with $x_{i j}$ in the $i j$ th position and let $\iota=(1, \ldots, 1)^{\prime}$ be $n \times 1$. We assume that the $n \times(m+1)$ matrix $X_{A}=(X, \iota)$ has full column rank. We estimate the components $f_{1}, \ldots, f_{m}$ in $(1.1)$ by penalized least squares by minimizing

$$
\sum_{i=1}^{n}\left\{y(i)-\sum_{j=1}^{m} f_{j}\left(x_{i j}\right)\right\}^{2}+\sum_{j=1}^{m} \lambda_{j}^{-1} \int_{0}^{1}\left\{f_{j}^{(2)}\right\}^{2} d x_{j}
$$

over $f_{j} \in F_{A}$, where the $\lambda_{j}$ are given positive constants and $f^{(2)}(x)=d^{2} f(x) / d^{2} x$. The integrals in (2.5) represent roughness penalties. Let $\hat{f}=\left(\hat{f}_{1}, \ldots, \hat{f}_{m}\right)$ minimize (2.5). Then each $\hat{f}_{j}$ is a cubic smoothing spline, that is $\hat{f}_{j}$ is a piecewise cubic. Spline smoothing is a popular statistical method of estimating a function when it is observed with noise; see Buja et al. (1989) for a discussion. Application of the backfitting algorithm is useful here because one dimensional smoothing is computationally straightforward, see for example Hutchinson and de Hoog (1985), whereas simultaneously smoothing all $m$ functions is far more difficult. To express the minimization of (2.5) geometrically define

$$
\langle g, h\rangle_{0 A}=\int_{0}^{1} g^{(2)}(x) h^{(2)}(x) d x .
$$

This makes $\langle g, h\rangle_{0 A}$ a semi-inner product with corresponding semi-norm $\|h\|_{O A}=$ $\langle h, h\rangle_{0 A}^{1 / 2}$. If $\|h\|_{0 A}=0$ then $h$ is linear. For $j=1, \ldots, m$, take $F_{j}=F_{A}$ and define the semi-inner products $\left\langle f_{j}, g_{j}\right\rangle_{j}=\lambda^{-1}\left\langle f_{j}, g_{j}\right\rangle_{0 A}$ and the linear functionals $\gamma_{i j}\left(f_{j}\right)=f_{j}\left(x_{i j}\right), i=1, \ldots, n$. Then (2.1) is a proper inner product for each $F_{j}$. Without further assumptions, however, (2.2) is only a semi-inner product for $F$ as $\|f\|_{F}$ can be zero with $f$ being nonzero. To see this note that if $\|f\|_{F}=0$, then each $f_{j}, j=1, \ldots, m$, is a linear function which we can write as $f_{j}(x)=\alpha_{j}+\beta_{j} x$, with $\alpha_{j}$ and $\beta_{j}$ constants. Because $\gamma_{i}(f)=\sum_{j} \gamma_{i j}\left(f_{j}\right)=0$ we have that $\sum_{j}\left(\alpha_{j}+\beta_{j} x_{i j}\right)=0$ for $i=1, \ldots, n$. By assumption the matrix $X_{A}$ is of full column rank so that $\beta_{1}, \ldots, \beta_{m}$ are zero and $\sum_{j} \alpha_{j}=0$. By choosing the $\alpha_{j}$ so that not all of them are zero but their sum is zero we obtain an $f \in F$ such that $\|f\|_{F}=0$ but $f \neq 0$. This degeneracy, which leads to multiple minima of $(2.5)$ is called the concurvity problem by Buja et al. (1989) and is fully dealt with in the next section. 
In most problems of practical interest it is easy to define the spaces $F_{j}$ so that Assumptions 2.1 and 2.2 are satisfied and hence Theorem 2.1 below holds. We now show two ways of doing so for the cubic spline smoothing problem. Let $F_{B}$ be the subspace of $F_{A}$ consisting of all functions $h$ such that $h(0)=0$, and define $F_{1}=F_{A}$ and $F_{j}=F_{B}$ for $j=2, \ldots, m$, with semi-inner products $\langle,\rangle_{O A}$ for all spaces. It can be readily checked that (2.2) is now a proper inner product. A second way of redefining the spaces $F_{j}$ is to take $F_{j}=F_{B}$ for $j=1, \ldots, m$ and define $F_{m+1}$ as the space of constant functions on $[0,1]$ with semi-inner product identically zero. Put $F=F_{1} \otimes F_{2} \otimes \cdots \otimes F_{m} \otimes F_{m+1}$ and minimize

$$
\sum_{i=1}^{n}\left\{y(i)-\sum_{j=1}^{m} f_{j}\left(x_{i j}\right)-\mu\right\}^{2}+\sum_{j=1}^{m} \lambda_{j}^{-1}\left\|f_{j}\right\|_{0 A}^{2}
$$

over $f_{1}, \ldots, f_{m} \in F_{B}$ and $\mu=f_{m+1}$. It can again be readily checked that

$$
\|f\|_{F}^{2}=\sum_{j=1}^{m}\left\|f_{j}\right\|_{0 A}^{2}+\sum_{i=1}^{n}\left\{\sum_{j=1}^{m} f_{j}\left(x_{i j}\right)+\mu\right\}^{2}
$$

defines a proper norm.

We now express the penalized least squares problem as an interpolation problem as in Weinert, Byrd and Sidhu (1980) and hence express the solution to (2.4) as a projection. Define the product space $H=\mathbb{R}^{n} \otimes F$ where $\mathbb{R}^{n}$ is $n$ dimensional Euclidean space. For convenience we will write $\epsilon=\left(\epsilon_{1}, \ldots, \epsilon_{n}\right)^{\prime}$ and denote a typical element of $H$ by $(\epsilon, f)$. Then define the linear functionals $\mu_{i}, i=1, \ldots, n$ on $H$ by $\mu_{i}(\epsilon, f)=\epsilon(i)+\gamma_{i}(f)$. It is now straightforward to check that $H$ is a Hilbert space with inner product

$$
\langle(\epsilon, f),(\xi, g)\rangle_{H}=\langle\epsilon, \xi\rangle_{0}+\sum_{j=1}^{m}\left\langle f_{j}, g_{j}\right\rangle_{0 j}+\sum_{i=1}^{n} \mu_{i}(\epsilon, f) \mu_{i}(\xi, g)
$$

and corresponding norm

$$
\|(f, \epsilon)\|_{H}^{2}=\|\epsilon\|_{0}^{2}+\sum_{j=1}^{m}\left\|f_{j}\right\|_{0 j}^{2}+\sum_{i=1}^{n}\left\{\mu_{i}(\epsilon, f)\right\}^{2}
$$

where $\langle\epsilon, \xi\rangle_{0}=\sum_{i} \epsilon_{i} \xi_{i}$. Assumption 2.1 guarantees that (2.7) is a proper inner product.

Next we show that (2.4) is equivalent to solving an interpolation problem whose solution is obtained as a projection. With the norm (2.8) the functional $\mu_{i}$ is bounded, and thus there exists a $\rho_{i} \in H$, called the representer of $\mu_{i}$, such that $\mu_{i}(\epsilon, f)=$ $\left\langle(\epsilon, f), \rho_{i}\right\rangle, i=1, \ldots, n$. Let $S$ be the subspace of $H$ generated by $\rho_{1}, \ldots, \rho_{n}$, and $P$ be the projection operator onto $S$. Further, let

$$
U=\left\{(\epsilon, f): \mu_{i}(\epsilon, f)=y(i), \quad i=1, \ldots, n\right\}
$$


and note that $U$ is nonempty because $(y, 0) \in U$, where $y=\{y(1), \ldots, y(n)\}^{\prime}$.

\section{LEMMA 2.1.}

(i) Solving (2.4) is equivalent to solving the interpolation problem

$$
\underset{(\epsilon, f) \in H}{\operatorname{minimize}}\|(\epsilon, f)\|_{H}: y(i)=\mu_{i}(\epsilon, f), \quad i=1, \ldots, n \text {. }
$$

(ii) For any $(\epsilon, f) \in H, \mu_{i} P(\epsilon, f)=\mu_{i}(\epsilon, f)$.

(iii) For any $(\epsilon, f) \in U, P(\epsilon, f)$ minimizes (2.9), and so the solution to (2.9), and hence the penalized least squares problem (2.4), exists (because $U$ is nonempty) and is unique.

(iv) If $\mu_{i}(\epsilon, f)=\mu_{i}(\xi, g), i=1, \ldots, n$, then $P(\epsilon, f)=P(\xi, g)$.

PROOF. For $(\epsilon, f) \in U$, the final term in (2.8) is $\sum_{i} y(i)^{2}$ and hence the solutions to (2.4) and (2.9) are the same. Parts (ii) and (iii) are immediate consequences of the projection theorem (see Weinert and Sidhu, 1978), and Part (iv) follows from Part (iii).

The backfitting algorithm applied to the penalized least squares problem proceeds as follows. Assign initial estimates $f_{1}^{(0)}, \ldots, f_{m}^{(0)}$ to $f_{1}, \ldots, f_{m}$; these can be the zero functions. At a typical point in the iteration suppose that we have the estimates $f_{1}^{(l)}, \ldots, f_{j-1}^{(l)}, f_{j}^{(l-1)}, \ldots, f_{m}^{(l-1)}$. We obtain $f_{j}^{(l)}$ by minimizing (2.4) with respect to $f_{j}$ keeping $f_{1}, \ldots, f_{j-1}, f_{j+1}, \ldots, f_{m}$ fixed at their current estimates. We continue the iteration until convergence. The corresponding algorithm for the interpolation problem initializes $f_{1}, \ldots, f_{m}$ as above and in addition initializes $\epsilon_{i}^{(0)}=y(i)-\gamma_{i}\left(f^{(0)}\right)$, $i=1, \ldots, n$. A typical step of the backfitting algorithm can be written as the solution to the penalized least squares problem

$$
\underset{f_{j} \in F_{j}}{\operatorname{minimize}} \sum_{i=1}^{n}\left\{y(i)-\mu_{i}(f)\right\}^{2}+\left\|f_{j}\right\|_{0 j}^{2}
$$

where $f_{l}$ is fixed for $l \neq j$.

To write the solution to (2.10) as a projection, we proceed exactly as for (2.4). Define the product space $H_{j}=\mathbb{R}^{n} \otimes F_{j}$ with typical element $\left(\epsilon, f_{j}\right)$, and define the linear functionals $\mu_{i j}$ in $H_{j}$ by $\mu_{i j}\left(\epsilon, f_{j}\right)=\epsilon_{i}+\gamma_{i j}\left(f_{j}\right), i=1, \ldots, n$. Then $H_{j}$ is a Hilbert space with inner product

$$
\left\langle\left(\epsilon, f_{j}\right),\left(\xi, g_{j}\right)\right\rangle_{j}=\sum_{i=1}^{n} \epsilon_{i} \xi_{i}+\left\langle f_{j}, g_{j}\right\rangle_{0 j}+\sum_{i=1}^{n} \mu_{i j}\left(\epsilon, f_{j}\right) \mu_{i j}\left(\xi, g_{j}\right)
$$

and corresponding norm

$$
\left\|\left(\epsilon, f_{j}\right)\right\|_{j}^{2}=\sum_{i=1}^{n} \epsilon_{i}^{2}+\left\|f_{j}\right\|_{0 j}^{2}+\sum_{i=1}^{n}\left\{\mu_{i j}\left(\epsilon, f_{j}\right)\right\}^{2} .
$$


The functionals $\mu_{i j}$ are bounded in $H_{j}$. Now let $\rho_{i j}$ be the representer of $\mu_{i j}$ in $H_{j}, S_{j}$ be the subspace of $H_{j}$ generated by $\rho_{1 j}, \ldots, \rho_{n j}$, and let $P_{j}$ be the projection operator onto $S_{j}$. Finally, for given $f_{l} \in F_{l}, l \neq j$, define the subset $U_{j}$ of $H_{j}$ by

$$
U_{j}=\left\{\left(\epsilon, f_{j}\right) \in H_{j}: \mu_{i j}\left(\epsilon, f_{j}\right)=y(i)-\sum_{l \neq j} \gamma_{i l}\left(f_{l}\right), \quad i=1, \ldots, n\right\}
$$

and note that $U_{j}$ is nonempty because $(y, 0) \in U_{j}$. The following lemma is just a special case of Lemma 2.1.

\section{LEMMA 2.2.}

(i) Solving (2.10) is equivalent to solving the interpolation problem

$$
\underset{\left(\epsilon, f_{j}\right) \in H_{j}}{\operatorname{minimize}}\|(\epsilon, f)\|_{j}^{2}: \mu_{i j}\left(\epsilon, f_{j}\right)=y(i)-\sum_{l \neq j} \gamma_{i l}\left(f_{l}\right), \quad i=1, \ldots, n
$$

(ii) For any $\left(\epsilon, f_{j}\right) \in H_{j}, \mu_{i j} P_{i}\left(\epsilon, f_{j}\right)=\mu_{i j}\left(\epsilon, f_{j}\right)$.

(iii) $P_{j}\left(\epsilon, f_{j}\right)$ minimizes (2.13) for any $\left(\epsilon, f_{j}\right) \in U_{j}$, and so the solution to (2.13), and hence the step of the backftting algorithm represented by the penalized least squares problem (2.10), exists (because $U_{j}$ is nonempty) and is unique.

Thus we have expressed the solutions to both the penalized least squares problem (2.4) and the steps of the backfitting algorithm (2.8) as projections in Hilbert spaces, but in different Hilbert spaces. To apply Theorem 1.1, we reexpress the steps of the backfitting algorithm as projections in $H$. First, for $j=1, \ldots, m$, write $P_{j}\left(\epsilon, f_{j}\right)=\left(\tilde{\epsilon}_{j}, \tilde{f}_{j}\right) \in H_{j}$, define the operator $T_{j}$ in $H$ by

$$
T_{j}(\epsilon, f)=\left(\tilde{\epsilon}, f_{1}, \ldots, f_{j-1}, \tilde{f}_{j}, f_{j+1}, \ldots, f_{m}\right)
$$

and the subset $M_{j}$ of $H$ by

$$
M_{j}=\left\{(\epsilon, f) \in H:\left(\epsilon, f_{j}\right) \in S_{j} \quad \text { in } \quad H_{j}\right\} .
$$

LEMMA 2.3 .

(i) Given $f_{l}, l \neq j$, suppose that $\epsilon$ and $f_{j}$ are such that $\left(\epsilon, f_{j}\right) \in U_{j}$, that is, $(\epsilon, f)=\left(\epsilon, f_{1}, \ldots, f_{m}\right) \in U$. Then $T_{j}(\epsilon, f)$ is the solution to the step of the backfiting algorithm given by (2.10), in the sense that $\tilde{\epsilon}$ and $\tilde{f}_{j}$ as defined in (2.14) are such that $\left(\tilde{\epsilon}, \tilde{f}_{j}\right) \in H_{j}$ solves (2.13) while $f_{l}$ is fixed for $l \neq j$.

(ii) The set $M_{j}$ is a Hilbert subspace of $H$ and $T_{j}$ is the projection operator onto $M_{j}(j=1, \ldots, m)$.

(iii) The space $S=M_{1} \cap \cdots \cap M_{m}$. 
The proof is given in the appendix.

Using Lemma 2.3, we can describe a typical cycle of the backfitting algorithm as taking $\left(\epsilon^{(l-1)}, f^{(l-1)}\right) \in U$ as estimates of $(\epsilon, f)$ from one step and applying to it the mapping $T=T_{m} T_{m-1} \cdots T_{1}$ to obtain the next estimate $\left(\epsilon^{(l)}, f^{(l)}\right) \in U$. Because $S=M_{1} \cap \ldots \cap M_{m}$, and $T_{j}$ is the projection operator onto $M_{j}, j=1, \ldots, m$ we obtain from Theorem 1.1,

\section{THEOREM 2.1 .}

(i) The penalized least squares problem (2.4) has a unique solution.

(ii) The linear operator $T^{N}$ converges strongly to the projection $P$ as $N \rightarrow \infty$, that is $\left\|\left(T^{N}-P\right)\left(\epsilon^{(0)}, f^{(0)}\right)\right\| \rightarrow 0$ as $N \rightarrow \infty$. Thus the backfitting algorithm applied to the penalized least squares problem converges to the unique optimum solution from any initial $f^{(0)}$, because by writing $\epsilon_{i}^{(0)}=y(i)-\mu_{i}\left(f^{(0)}\right)$, $i=1, \ldots, n$, we have $\left(\epsilon^{(0)}, f^{(0)}\right) \in U$.

It is straightforward to extend our results to the case where some functionals are observed without error. Thus suppose that

$$
y(i)=\gamma_{i}(f)=\sum_{j} \gamma_{i j}\left(f_{j}\right) \quad(i=n+1, \ldots, n+r)
$$

and $f$ is estimated by minimizing (2.4) subject to (2.16). Define $\mu_{i}(\epsilon, f)=\gamma_{i}(f)$, $i=n+1, \ldots, n+r$, and replace $(2.8)$ by

$$
\|(\epsilon, f)\|_{H}^{2}=\|\epsilon\|_{0}^{2}+\sum_{j=1}^{m}\left\|f_{j}\right\|_{0 j}^{2}+\sum_{i=1}^{n+r}\left\{\mu_{i}(\epsilon, f)\right\}^{2} .
$$

Then Theorem 2.1 still holds. We illustrate the usefulness of this extension by two examples.

EXAMPLE 2.2. Suppose that in Example (2.1) we know that the first component $f_{1}$ is periodic so that $f_{1}(0)=f_{1}(1)$ and $f_{1}^{(1)}(0)=f_{1}^{(1)}(1)$. Define the functionals $\gamma_{n+1}(f)=f_{1}(0)-f_{1}(1)$ and $\gamma_{n+2}(f)=f_{1}^{(1)}(0)-f_{1}^{(1)}(1)$ and the extra two observations $y(n+1)=\gamma_{n+1}(f)$ and $y(n+2)=\gamma_{n+2}(f)$ with $y(n+1)=y(n+2)=0$. Then the estimate of $f_{1}$ is a periodic cubic spline. Periodic cubic splines are discussed by Cogburn and Davis (1974) and Wahba (1980). Both these papers use periodic splines to estimate the spectral density of a stationary process.

EXAMPLE 2.3. Suppose that in Example 2.1 the curves $f_{1}$ and $f_{2}$ coincide for $x \leq x^{*}$ and for $x>x^{*}$ the curve $f_{2}$ branches out from $f_{1}$. For example $f_{1}$ may be a control curve and $f_{2}$ a treatment curve with the treatment applied at time $x=x^{*}$ so that $f_{1}$ and $f_{2}$ are distinct for $x>x^{*}$. We assume that at $x=x^{*}$ the two curves 
coincide so that $f_{1}\left(x^{*}\right)=f_{2}\left(x^{*}\right)$. To enforce this restriction define the functional $\gamma_{n+1}(f)=f_{1}\left(x^{*}\right)-f_{2}\left(x^{*}\right)$ and let $y(n+1)=\gamma_{n+1}(f)$ with $y(n+1)=0$. The functions $f_{1}$ and $f_{2}$ are now estimated as above. Silverman and Wood (1987) and Kohn and Ansley (1991) discuss the estimation of such branching curves by spline smoothing and give a number of examples.

\section{Degenerate Case}

Although the results in Section 2 are sufficient to solve most problems of practical interest, it is sometimes computationally convenient to relax Assumption 2.1 so that (2.2) is a semi-inner product in $F$. In this case (2.4) no longer has a unique solution and this is called the concurvity problem by Buja et al. (1989). For instance, in Example 2.1 we may want to take each $F_{j}$ as $F_{A}$ (the space of functions with square integrable second derivatives) because the algorithm we have available carries out unconstrained cubic spline smoothing. We showed in Example 2.1 that Assumption 2.1 does not hold with this choice of $F_{j}$. We now show that as long as (2.1) is a proper inner product for each space $F_{j}(j=1, \ldots, m)$ then the backfitting algorithm still converges to a solution of (2.4) and if $\hat{f}$ and $\hat{g}$ are two solutions then $\|\hat{f}-\hat{g}\|_{F}=0$.

We replace Assumptions 2.1 and 2.2 with

ASSUMPTION 3.1. For $f_{j} \in F_{j}$, if $\left\|f_{j}\right\|_{0 j}=0$ and $\gamma_{i j}\left(f_{j}\right)=0$ for $i=1, \ldots, n$, then $f_{j}=0$ for $j=1, \ldots, m$. This is sufficient to ensure that $(2.1)$ is an inner product on $F_{j}$.

ASSUMPTION 3.2. $F_{j}$ is a Hilbert space with inner product $(2.1)(j=1, \ldots, m)$.

Now Assumptions 3.1 and 3.2 are not sufficient to ensure that (2.2) is a proper inner product on $F$. In general, it is only a semi-inner product. We accommodate this problem by considering the space $F^{*}$ of equivalence classes of elements of $F$, where we say that $f, g \in F$ are equivalent if $\|f-g\|_{F}=0$. Let $[f]$ be the equivalence class generated by $f$. If $[f]=[g]$ then

$$
\left\|f_{j}-g_{j}\right\|_{0 j}=0 \quad(j=1, \ldots, m) \quad \text { and } \quad \gamma_{i}(f)=\gamma_{i}(g) \quad(i=1, \ldots, n) .
$$

It is clear that if $\hat{f}$ is a solution to (2.4) then so are all members of its equivalence class.

If $[f]=[g]$ then $\gamma_{i}(f)=\gamma_{i}(g)(i=1, \ldots, n)$ showing that the linear functionals $\gamma_{i}$ are well defined in $F^{*}$, and $F^{*}$ is a Hilbert space with inner product (2.2). We now show that the results in Section 2 apply to the elements of $F^{*}$ rather than $F$. First we define the space $H^{*}=\mathbb{R}^{n} \otimes F^{*}$ whose elements are equivalence classes of 
elements in $H$. We write a typical element of $H^{*}$ as $(\epsilon,[f])$ so that $(\epsilon,[f])=(\epsilon,[g])$ if $[f]=[g]$. This means that (2.7) and (2.8) are a proper inner product and norm, respectively, in $H^{*}$. Let $U^{*}$ be defined with respect to $H^{*}$ in the same way that $U$ is defined with respect to $H$. Then Lemma 2.1 holds without modification while Lemma 2.2 concerns $H_{j}=\mathbb{R}^{n} \otimes F_{j}$ and is not affected. To check that Lemma 2.3 holds, we first show that the linear operator $T_{j}$ in (2.14) is well defined in $H^{*}$. This is obtained from the following lemma.

LEMMA 3.1. Suppose that $z=\left(z_{1}, \ldots, z_{m}\right) \in F$ and $[z]=[0]$. Then $P_{j}\left(0, z_{j}\right)=$ $\left(0, z_{j}\right)$ in $H_{j}, j=1, \ldots, m$.

PROOF. Let $y_{i j}=\gamma_{i j}\left(z_{j}\right), i=1, \ldots, n, j=1, \ldots, m$. Because $[z]=[0]$, $\left\|z_{j}\right\|_{0 j}=0$, and thus $\left(0, z_{j}\right)$ is the solution to the minimization problem

$$
\underset{\left(\epsilon, f_{j}\right) \in H_{j}}{\operatorname{minimize}}\left\|\left(\epsilon, f_{j}\right)\right\|_{j}^{2}: \mu_{i j}\left(\epsilon, f_{j}\right)=y_{i j}, \quad i=1, \ldots, n .
$$

By Lemma 2.1 the solution is unique and $P_{j}\left(0, z_{j}\right)=\left(0, z_{j}\right), j=1, \ldots, m$.

For $z \in F$, if $[z]=[0]$, then $T_{j}(0, z)=(0, z)$ by Lemma 3.1. Hence if $f, g \in F$ are equivalent, then $T_{j}(\epsilon, f)=T_{j}(\epsilon, g)+(0, f-g)$ showing that $T_{j}(\epsilon, f)$ and $T_{j}(\epsilon, g)$ are equivalent. This shows that $T_{j}$ is well defined in $H^{*}$. Let $M_{j}^{*}$ be the range of the projection $T_{j}^{*}$. Then Lemma (2.3) holds in $H^{*}$ if $M_{j}$ is replaced by $M_{j}^{*}$.

The operator $T$ and the projection operator $P$ are well defined in $H^{*}$. We can deduce the following results from Theorem (2.1). If $(\epsilon, f) \in U$ then any member of the equivalence class $P(\epsilon,[f])$ minimizes $(2.4)$ and if $(\hat{\epsilon}, \hat{f})$ and $(\hat{\epsilon}, \hat{g})$ belong to $P(\epsilon,[f])$ then $[\hat{f}-\hat{g}]=[0]$. The iterates $T^{N}(\epsilon,[f])$ converge to $P(\epsilon,[f])$ so that $\left\|T^{N}(\epsilon, f)-(\hat{\epsilon}, \hat{f})\right\|_{H} \rightarrow 0$ as $N \rightarrow \infty$, where $(\hat{\epsilon}, \hat{f})$ is a solution to (2.4). This means that $\left\|T^{N}(\epsilon, f)\right\|_{H}$ is nonincreasing and tends to $\|(\hat{\epsilon}, \hat{f})\|_{H}$. Now, for any $N \geq 0$ either $T^{N+1}(\epsilon, f)=T^{N}(\epsilon, f)$ in which case we have converged, or $\left\|T_{j} T^{N}(\epsilon, f)\right\|_{H}<$ $\left\|T^{N}(\epsilon, f)\right\|_{H}$ for some $j$ in which case $\left\|T^{N+1}(\epsilon, f)\right\|_{H}<\left\|T^{N}(\epsilon, f)\right\|_{H}$. This shows that $T^{N}(\epsilon, f)$ converges to a unique element of $F$. Finally, if $z \in F$ and $[z]=0$ then $T(0, z)=(0, z)$ so that if $[f]=[g]$ then $T^{N}(\epsilon, f)=T^{N}(\epsilon, g)+(0, f-g)$ showing that the separation between $f$ and $g$ is preserved by $T^{N}$. This discussion is summarized in the following theorem.

THEOREM 3.1. Suppose Assumptions 3.1 and 3.2 hold. Then

(i) the penalized least squares problem (2.4) has a solution that is unique up to equivalence in $F^{*}$, that is, up to a displacement $z=\left(z_{1}, \ldots, z_{m}\right)$ such that $\left\|z_{j}\right\|_{0 j}=0, j=1, \ldots, m$ and $\gamma_{i}(z)=0, i=1, \ldots, n$.

(ii) The linear operator $T^{N}$ in $H^{*}$ converges strongly to the projection $P$ as $N \rightarrow \infty$, that is $\left\|\left(T^{N}-P\right)(\epsilon,[f])\right\| \rightarrow 0$ as $N \rightarrow \infty$ for $(\epsilon, f) \in U \subset H$. 
(iii) The backfitting algorithm converges to a unique element of $F$ which is an optimum solution from any initial value $f \in F$. Equivalent solutions can be found from any optimum solution by displacements as in (i).

The following corollary can be immediately deduced from Theorem 3.1 and is Theorem 9 of Buja et al. (1989).

COROLLARY 3.1. Suppose that the functionals $\mu_{i j}$ are the evaluation functionals $\mu_{i j}\left(f_{j}\right)=f_{j}\left(x_{i j}\right), j=1, \ldots, m$ and $i=1, \ldots, n$. Let $f_{j}=\left\{f_{j}\left(x_{1 j}\right), \ldots, f_{j}\left(x_{n j}\right)\right\}^{\prime}$, and let $\left\|f_{j}\right\|_{0 j}=f_{j}^{\prime} A_{j} f_{j}$ where $A_{j}$ is a positive-semidefinite matrix, $j=1, \ldots, m$. Assume that Assumption 3.1 holds. Then Theorem 3.1 holds.

EXAMPLE 2.2. (continued). Consider again the additive model (1.1) estimated by cubic splines with no restrictions on initial conditions so that $F_{j}=F_{A}$ for all $j$. To satisfy Assumptions 3.1 and 3.2, we require only that the vector $X_{j}=\left(x_{1 j}, \ldots, x_{n j}\right)^{\prime}$ is linearly independent of the vector $\iota=(1, \ldots, 1)^{\prime}$ for each $j=1, \ldots, m$, so that each step of each iteration of the backfitting procedure has a unique solution. Then, from any set of starting values, the backfitting algorithm converges to a unique solution $\hat{f}=\left(\hat{f}_{1}, \ldots, \hat{f}_{m}\right)^{\prime}$ in $F$, with each $\hat{f}_{j}$ a cubic spline. From Example 2.1 and the discussion above, there are multiple solutions equivalent to $f$ and given by $\hat{f}+z$ with $[z]=[0]$. Let $z=\left(z_{1}, \ldots, z_{m}\right) \in F$. We showed in Section 2 that if $[z]=[0]$ then $z_{j}(x)=\alpha_{j}+\beta_{j} x_{j}$ with the constants $\alpha_{j}$ and $\beta_{j}$ constrained by $\sum_{j}\left(\alpha_{j}+\beta_{j} x_{j}\right)=0$ for $j=1, \ldots, m$. In particular $[z]=[0]$ if we take all the $\beta_{j}$ to be zero and $\sum_{j} \alpha_{j}=0$ without all the $\alpha_{j}$ s being zero.

Our results also show that we can introduce redundant components into the model and still have convergence. For example, suppose that $x_{i j}=a+b x_{i j^{\prime}}, j \neq j^{\prime}$ and $i=1, \ldots, n$. Although one of $f_{j}$ and $f_{j^{\prime}}$ is redundant because the knots for the $j$ th component are a linear combination of those for the $j^{\prime}$ th component, the backfitting algorithm will still converge to the estimates $\hat{f}_{j}$ and $\hat{f}_{j^{\prime}}$ of $f_{j}$ and $f_{j^{\prime}}$ determined by the initial conditions. It is sometimes faster to apply the backfitting algorithm to subsets of components rather than individual components in which case the functionals $\gamma_{i j}$ will not be evaluation functionals. An important application of this observation is smoothing with time series structural component models.

EXAMPLE 3.1. Time series structural component model. Suppose that we have quarterly data collected in time order so that

$$
y(i)=t(i)+s(i)+f_{2}\left(x_{i 2}\right)+e(i), \quad i=1, \ldots, n
$$

where $t(i)$ is the trend, $s(i)$ is the seasonal, and $f_{2}\left(x_{i 2}\right)$ is a function of a regressor of 
interest. We define the semi-norms

$$
\|t\|_{0 t}^{2}=\sum_{i=1}^{n}\{t(i)-t(i-1)\}^{2}, \quad\|s\|_{0 s}^{2}=\sum_{i=1}^{n}\{s(i)-s(i-4)\}^{2} .
$$

Following Kitagawa and Gersch (1984) we estimate $t, s$ and $f_{2}$ by minimizing

$$
\sum_{i=1}^{n}\left\{y(i)-t(i)-s(i)-f_{2}\left(x_{i 2}\right)\right\}^{2}+\|t\|_{0 t}^{2}+\|s\|_{0 s}^{2}+\left\|f_{2}\right\|_{0 A}^{2}
$$

over $t=\{t(0), \ldots, t(n)\} \in \mathbb{R}^{n+1}, s=\{s(-3), \ldots, s(n)\}^{\prime} \in \mathbb{R}^{n+4}$, and $f_{2} \in F_{A}$. Although we can minimize (3.1) by applying the backfitting algorithm to the individual components $t, s$, and $f_{2}$, it is computationally more efficient to form the composite component $f_{1}(i)=\{t(i), s(i)\}$, define the functionals $\gamma_{i 1}\left(f_{1}\right)=t(i)+s(i)$ and $\gamma_{i 2}\left(f_{2}\right)=f_{2}\left(x_{i 2}\right), i=1, \ldots, n$ and apply the backfitting algorithm to the two component model $y(i)=\gamma_{1 i}\left(f_{1}\right)+f_{2}\left(x_{i 2}\right)+e(i)$. Thus $t$ and $s$ are estimated simultaneously as in Kitagawa and Gersch (1984). We note that the functionals $\gamma_{i 1}$ are not evaluation functionals.

\section{Appendix: Proofs}

We now prove Lemma 2.3 and Theorem 1.1. Halperin (1962) proves a stronger result than Theorem 1.1 but if we look carefully at his argument we obtain a very simple proof of Theorem 1.1 which we present for completeness.

PROOF OF LEMMA 2.3. Part (i) follows immediately from Lemma 2.2 and the definition of $T_{j}$. For Part (ii), note first that because $P_{j}$ is a projection operator in $H_{j}, T_{j}$ is a linear operator in $H$ and $M_{j}=\left\{(\epsilon, f) \in H: T_{j}(\epsilon, f)=(\epsilon, f)\right\}$, so that $M_{j}$ is a Hilbert subspace of $H$. Now by (2.14), for any $(\epsilon, f) \in H, T_{j}(\epsilon, f) \in M_{j}$ and $(\epsilon, f)-T_{j}(\epsilon, f)=\left(\epsilon-\tilde{\epsilon}, 0, \ldots, f_{j}-\tilde{f}_{j}, 0, \ldots, 0\right)$. Hence for any $(\xi, g) \in M_{j}$,

$$
\left\langle(\epsilon, f)-T_{j}(\epsilon, f),(\xi, g)\right\rangle=\left\langle\left(\epsilon, f_{j}\right)-P_{j}\left(\epsilon, f_{j}\right),\left(\xi, g_{j}\right)\right\rangle_{j}=0
$$

so that $(\epsilon, f)-T_{j}(\epsilon, f) \in M_{j}^{\perp}$, the orthogonal complement of $M_{j}$, and $T_{j}$ is the projection operator onto $M_{j}$.

To show that $S=M_{1} \cap \cdots \cap M_{m}$, note first that by (2.14) and Lemma 2.2, for any $(\epsilon, f) \in H, \mu_{i} T_{j}(\epsilon, f)=\mu_{i}(\epsilon, f), i=1, \ldots, n$, and hence by Lemma 2.1 $P T_{j}(\epsilon, f)=P(\epsilon, f)$. Thus for $(\epsilon, f) \in M_{j}^{\perp}, P(\epsilon, f)=P T_{j}(\epsilon, f)=0$, so that $(\epsilon, f) \in S^{\perp}$, and $M_{j}^{\perp} \subset S^{\perp}, j=1, \ldots, m$. Hence $S \subset M_{1} \cap \cdots \cap M_{m}$.

To complete the proof, we show that $S^{\perp} \subset M_{1}^{\perp}+\cdots+M_{m}^{\perp}$. The required result follows because $M_{1}^{\perp}+\cdots+M_{m}^{\perp} \subset\left(M_{1} \cap \cdots \cap M_{m}\right)^{\perp}$. Take $(\epsilon, f) \in S^{\perp}$. Then

$$
\epsilon_{i}+\gamma_{i 1}\left(f_{1}\right)+\cdots+\gamma_{i m}\left(f_{m}\right)=0 \quad(i=1, \ldots, n) .
$$


For $j=1, \ldots, m$, define the element $\left(\epsilon^{(j)}, f^{(j)}\right) \in H$ such that $f_{j}^{(j)}=f_{j}, f_{l}^{(j)}=0$ $(l \neq j)$ and $\epsilon_{i}^{(j)}=-\gamma_{i j}\left(f_{j}\right), j=1, \ldots, n$, so that $\left(\epsilon^{(j)}, f_{j}^{(j)}\right) \in S_{j}^{\perp}$ in $H_{j}$ and hence $\left(\epsilon^{(j)}, f^{(j)}\right) \in M_{j}^{\perp}$ in $H$. By (A.1), $(\epsilon, f)=\sum_{j}\left(\epsilon^{(j)}, f^{(j)}\right)$, so that $(\epsilon, f) \epsilon$ $M_{1}^{\perp}+\ldots+M_{m}^{\perp}$.

PROOF OF THEOREM 1.1. The proof of Theorem 1.1 depends on the following three results.

(i) $S=\{x: T x=x\}$ and for any $x \in H$, either $T x=x$ or $\|T x\|<\|x\|$.

(ii) If $y \in \overline{R(I-T)}$, where $R(I-T)$ is the range of $I-T$, then $T^{j} y \rightarrow 0$ as $j \rightarrow \infty$.

(iii) $S^{\perp} \subset \overline{R(I-T)}$.

It follows from (iii) that we can write any $h \in H$ as $h=x+y$ with $x \in S$ and $y \in \overline{R(I-T)}$ so that from (i) and (ii), $T^{N} h=x+T^{N} y \rightarrow x$ as $N \rightarrow \infty$.

To show that (i) holds we note that for any $x \in H$ either $T_{j} x=x$ for $j=1, \ldots, m$, in which case $T x=x$ and $x \in S$, or $\left\|T_{j} x\right\|<\|x\|$ for at least one $j$, and then $\|T x\|<\|x\|$ and $x \notin S$. We show that (ii) holds for $y \in R(I-T)$ as the extension to its closure is straightforward. We show below that

$$
\|x-T x\|^{2} \leq m\left(\|x\|^{2}-\|T x\|^{2}\right) .
$$

It follows that

$$
\left\|T^{j} x-T^{j+1} x\right\|^{2} \leq m\left(\left\|T^{j} x\right\|^{2}-\left\|T^{j+1} x\right\|^{2}\right) .
$$

Because $\left\|T^{j} x\right\|$ is a nonincreasing sequence for a given $x$, it follows that $\left\|T^{j} x\right\|^{2}-$ $\left\|T^{j+1} x\right\|^{2} \rightarrow 0$ as $j \rightarrow \infty$, and so $T^{j}(I-T) x \rightarrow 0$ implying that (ii) holds. To show that (A.2) holds, it is sufficient to consider the case $m=2$ and write

$$
x-T x=\left(x-T_{1} x\right)+\left(T_{1} x-T_{2} T_{1} x\right)
$$

so that

$$
\|x-T x\|^{2} \leq\left(\left\|x-T_{1} x\right\|+\left\|T_{1} x-T_{2} T_{1} x\right\|\right)^{2} \leq 2\left(\left\|x-T_{1} x\right\|^{2}+\left\|T_{1} x-T_{2} T_{1} x\right\|^{2}\right)
$$

Because $T_{1}$ and $T_{2}$ are projections, $\left\|x-T_{1} x\right\|^{2}=\|x\|^{2}-\left\|T_{1} X\right\|^{2}$ and $\left\|T_{1} x-T_{2} T_{1} x\right\|^{2}=$ $\left\|T_{1} x\right\|^{2}-\left\|T_{2} T_{1} x\right\|^{2}$ and (A.2) follows.

We show that $R(I-T)^{\perp} \subset S$ from which (iii) follows. If $x \in R(I-T)^{\perp}$ then $\langle x,(I-T) x\rangle=0$ so that $T x=x$. 


\section{References}

Buja, A., Hastie, T. \& Tibshirani, R. (1989), 'Linear smoothers and additive models (with discussion)', Ann. Statist. 17, 453-555.

Cogbum, R. \& Davis, H. T. (1974), 'Periodic spline and spectral estimation', Ann. Statist. 2, 1108-1126.

Elfving, T. (1980), 'Block-iterative methods for consistent and inconsistent linear equations', Numer. Math. 35, 1-12.

Friedman, J. H. \& Stuetzle, W. (1981), 'Projection pursuit regression', J. Amer. Statist. Assoc. 76, 817-823.

Halperin, I. (1962), 'The product of projection operators', Acta Sci. Math. 23, 96-99.

Hutchinson, M. F. \& de Hoog, F. R. (1985), 'Smoothing noisy data with spline functions', Numer. Math. 47, 99-106.

Kaczmarz, S. (1937), 'Angenaherte auflosung von systemen linearer gleichungen', Bull. Acad. Polon. Sci. pp. 335-357.

Kitagawa, G. \& Gersch, W. (1984), 'A smoothness priors-state space modeling of time series with trend and seasonality', J. Amer. Statist. Assoc. 79, 378-389.

Kohn, R. \& Ansley, C. F. (1991), 'A signal extraction approach to the estimation of treatment and control curves', J. Amer. Statist. Assoc. 86, 1034-1041.

von Neumann, J. (1950), Functional operators Vol. II: The Geometry of Orthogonal Spaces, Vol. 22 of Ann. of Math. Stud., Princeton University Press, Princeton.

Silverman, B. W. \& Wood, J. T. (1987), 'The nonparametric estimation of branching curves', J. Amer. Statist. Assoc. 82, 551-558.

Wahba, G. (1980), 'Automatic smoothing of the log periodogram', J. Amer. Statist. Assoc. 75, 122-132.

Wecker, W. E. \& Ansley, C. F. (1982), 'Nonparametric multiple regression by the alternating projection method', in Proc. ASA Bus. Econ. Statist. Section, pp. 311-316.

Weinert, H. L. \& Sidhu, G. S. (1978), 'A stochastic framework for recursive computation of spline functions: Part I, Interpolating splines', IEEE Trans. Inform. Theory 24, 45-50.

Weinert, H. L., Byrd, R. H. \& Sidhu, G. S. (1980), 'A stochastic framework for recursive computation of spline functions: Part II, Smoothing splines', J. Optim. Theory Appl. 30, 255-268.

Department of Accounting and Finance

University of Auckland

Private Bag, Auckland

New Zealand

\section{Australian Graduate School of Management University of NSW \\ Kensington}

New South Wales

Australia 